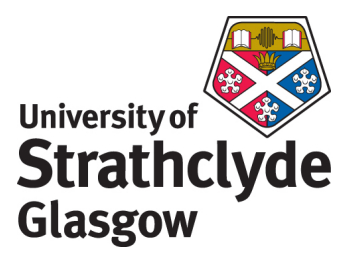

Elgamel, Sherif Abd el halim and Soraghan, J.J. (2009) Target tracking enhancement using a Kalman filter in the presence of interference. In: Geoscience and Remote Sensing Symposium, 2009 IEEE International, IGARSS 2009, 12-17 July 2009, Cape Town, South Africa.

http://strathprints.strath.ac.uk/27160/

Strathprints is designed to allow users to access the research output of the University of Strathclyde. Copyright $(C$ and Moral Rights for the papers on this site are retained by the individual authors and/or other copyright owners. You may not engage in further distribution of the material for any profitmaking activities or any commercial gain. You may freely distribute both the url (http://strathprints.strath.ac.uk) and the content of this paper for research or study, educational, or not-for-profit purposes without prior permission or charge. You may freely distribute the url (http://strathprints.strath.ac.uk) of the Strathprints website.

Any correspondence concerning this service should be sent to The Strathprints Administrator: eprints@cis.strath.ac.uk 


\title{
TARGET TRACKING ENHANCEMENT USING A KALMAN FILTER IN THE PRESENCE OF INTERFERENCE
}

\author{
Sherif A Elgamel and John Soraghan \\ Department of Electronic and Electrical Engineering, University of Strathclyde \\ phone: +(44) 1415482514,e-mail: sherifelgamel73@gmail.com, j.soraghan@eee.strath.ac.uk
}

\begin{abstract}
In this paper we present a new target tracking enhancement system that uses a Kalman filter in the presence of interference. If the radar (seeker) is affected by different types of interference, this will affect the missile trajectory towards the target and may cause inaccurate tracking. In the new system a six-state Kalman filter is utilized to perform the tracking task and to carry out smoothing to the corrupted trajectory. This also provides good information about the target velocity in three dimensions which is very important information about the target.

A three dimensional scenario between target (with high manoeuvre) and missile is used to illustrate the performance of the system in the case when (i) no interference is present and (ii) interference is present. The performance of the filtered trajectory using the Kalman tracker will be assessed for different guidance methods: including (i) proportional navigation (ii) pure pursuit and (iii) constant bearing. The Kalman improvement for the tacking for the three guidance method will be analysed.
\end{abstract}

Index Terms- Tracking, Kalman Filter, Interference

\section{INTRODUCTION}

Monopulse seekers are commonly used in target tracking because of their angular accuracy [1]. These types of seekers provide a better angular accuracy and less sensitivity to fluctuation in the radar cross section (RCS) of the target compared with other types of tracking radars (such as sequential lobbing and conical scan) [2]. Historically, monopulse seekers (or radars) employ two separate feed horns on a single antenna element in order to generate two receive beams that are slightly offset in azimuth (or elevation) angle. Sum and difference outputs are formed by summing and subtracting the two beam outputs, respectively. The ratio of difference to sum output voltages, called the error voltage, is then used to determine the degree of correction necessary to realign the beam axis with the target. With the introduction of phased array technology, it became unnecessary to employ special hardware for monopulse processing, since the array itself can electronically form the multiple beams needed [3, 4]. A typical conventional monopulse processor for phased array radar is obtained by appropriately phasing the individual array channels to obtain sum and difference outputs. The ratio of difference to sum outputs provides the measure by which the angle offset from the beam axis (i.e., look direction) is determined. The updated angle measurement is used to compute phases for the channels so as to realign the beam axis with the target. The radar (seeker) is affected by different types of interference [5]; this will affect the missile trajectory towards the target and may cause inaccurate tracking. Using a Kalman filter performs the tracking task and facilitates smoothing of the corrupted trajectory. This also provides good information about the target velocity in three dimensions.

This paper is organized as follows. Section 2 discusses the basic structure of the proposed Kalman filter. The target missile general kinematic equations are discussed in section 3. Section 4 presents the simulation model of the missile and target trajectories using three homing guidance methods namely; (i) proportional navigation, (ii) pure pursuit, and (iii) constant bearing with interference. Section 5 describes the Kalman Filter based enhancement on the interfered missile target trajectory. A set of simulation results is presented in section 6 for a series of 3-dimensional scenarios. Section 7 is the conclusion of this paper

\section{BASIC STRUCTURE OF THE PROPOSED KALMAN FILTER}

The Kalman filter is a linear estimator that minimizes the mean squared error as long as the target dynamics are modelled accurately. All other recursive filters, such as the $\alpha \beta \gamma$ and the Benedict-Bordner filters [6], are special cases of the general solution provided by the Kalman filter for the mean squared estimation problem. The filtering equation is [7]

$$
x(n \backslash n)=x_{s}(n)=x(n \backslash n-1)+K(n)[y(n)-G x(n \backslash n-1)]
$$

The measurement vector is

$$
y(n)=G x(n)+v(n)
$$

where $v(n)$ is zero mean, white Gaussian noise with covariance $\Re_{c}$

$$
\Re_{c}=E\left\{y(n) y^{t}(n)\right\}
$$

The gain (weight) vector is dynamically computed as

$$
K(n)=P(n \backslash n-1) G^{t}\left[G P(n \backslash n-1) G^{T}+\Re_{c}\right]^{-1}
$$

where the measurement noise matrix $P$ represents the predictor covariance matrix, and is equal to

$$
P(n+1 \backslash n)=\Phi P(n \backslash n) \Phi^{t}+Q
$$

where $Q$ is the covariance matrix for the input $u$,

$$
Q=E\left\{u(n) u^{t}(n)\right\}
$$


and $\Phi$ is the diagonal transmission matrix. The corrector equation (covariance of the smoothed estimate) is

$$
P(n \backslash n)=[I-K(n) G] P(n \backslash n-1)
$$

Finally, the predictor equation is

$$
x(n+1 \backslash n)=\Phi x(n \backslash n)
$$

\section{GENERAL KINEMATIC EQUATIONS OF MISSILE TARGET ENGAGEMENT}

The mathematical algorithm, that describes the target-missile engagement, uses a kinematic modelling method, in which the missile is assumed to be a massless point and the equations describing its motion are derived without considering the causes to this motion. In this algorithm a time step update for both the target and missile velocity and position in the three dimension space are calculated according to the ideal bonds equations (equations of the law of guidance). These are dependent upon the method of guidance used to guide the missile for intercepting the target. The Kalman tracker inside the missile processor filters the corrupted target position for each time-step. The filtered values are used to calculate the next position for the missile towards the target. This is continued until the line of site (LOS) distance between the missile and the target equals zero or equal the fusing distance of the missile.

Utilizing this method, it is possible to determine the shape of trajectories and the necessary manoeuvrability of the missile under the assumption that the missile velocity is a known function of time (which is determined from Kalman tracker) [8]. The position of the missile w.r.t target is determined in the polar coordinate system by radius vector $\bar{D}$, as shown in Fig 1.

The principle law of motion of a self-guidance missile in the vector form is given by [9],

$$
\frac{d \bar{D}}{d t}=\bar{V}_{T}-\bar{V}_{M}
$$

where $T$ and $M$ denotes the target and missile respectively, $\bar{D}$ is the vector of line of sight (LOS) length, $\bar{V}_{T}$ is the vector of target velocity with angles $\theta_{T}$ and $\varphi_{T}$, and $\bar{V}_{M}$ is the vector of missile velocity with angles $\theta_{M}$ and $\varphi_{M}$. The scalar Kinematic equations of missile centre of gravity motion are [8]:

$$
\begin{aligned}
& \dot{D}=V_{T} \cos \left(\sigma-\varphi_{T}\right) \cos \left(\varepsilon-\theta_{T}\right)-V_{M} \cos \left(\sigma-\varphi_{M}\right) \cos \left(\varepsilon-\theta_{M}\right) \\
& D \dot{\varepsilon}=V_{M} \cos \left(\sigma-\varphi_{M}\right) \sin \left(\varepsilon-\theta_{M}\right)-V_{T} \cos \left(\sigma-\varphi_{T}\right) \sin \left(\varepsilon-\theta_{T}\right) \\
& D \cos \varepsilon \dot{\sigma}=V_{M} \sin \left(\sigma-\varphi_{M}\right) \cos \theta_{M}-V_{T} \sin \left(\sigma-\varphi_{T}\right) \cos \theta_{T}
\end{aligned}
$$

In our simulations, for a typical case of radar guided missile, three homing guidance methods; (i) proportional navigation, (ii) pure pursuit, and (iii) constant bearing are used [8].

\section{EFFECT OF INTERFERENCE ON THE MISSILE TARGET ENGAGEMENT}

To evaluate the effect of the interference on the missile target engagement we calculate (i) the unfiltered position of the target $X_{T_{t(i+1)}}, Y_{T_{t(i+1)}}, Z_{T_{t(i+1)}}$ due to interference by adding

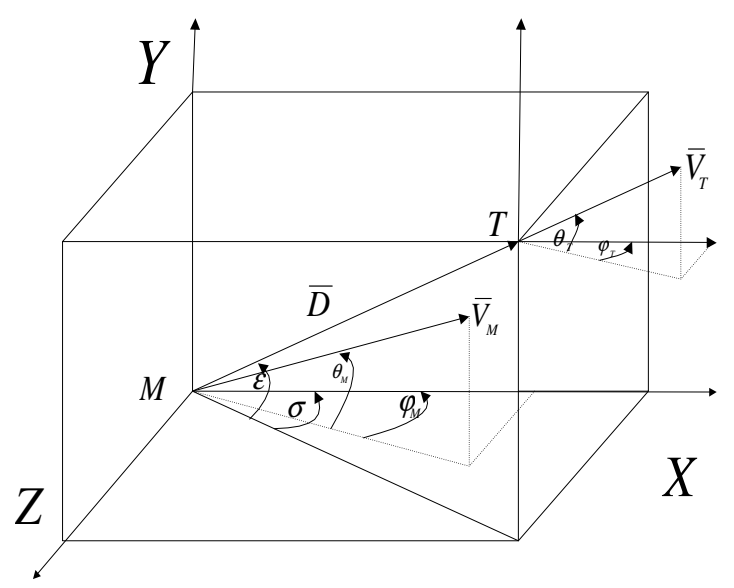

Fig 1- Geometry of homing guidance system

the presence noise error to the real target position $X_{T_{(i+1)}}, Y_{T_{(i+1)}}, Z_{T_{(i+1)}}$ and (ii) the missile unfiltered position $X_{M_{L U}}, Y_{M_{L}}, Z_{M_{L(1)}}$, then the LOS angles under interference $\varepsilon_{I_{(i+1)}}, \sigma_{I_{(i+1)}}$ can be calculated from the following equations

$$
\begin{gathered}
\varepsilon_{I i+1}=\tan ^{-1}\left(\frac{Y_{T_{I_{i+1}}}-Y_{M_{I_{i}}}}{\sqrt{\left(X_{T_{I i+1}}-X_{M_{I_{i}}}\right)^{2}+\left(Z_{T_{I_{i+1}}}-Z_{M_{I_{i}}}\right)^{2}}}\right) \\
\sigma_{I i+1}=\tan ^{-1}\left(\frac{Z_{T_{I_{i+1}}}-Z_{M_{I_{i}}}}{X_{T_{I_{i+1}}}-X_{M_{I_{i}}}}\right)
\end{gathered}
$$

This process is repeated along the target and missile flights until the difference between the angles $\varepsilon_{I_{i}}, \mathcal{E}_{I_{u+1}}$ becomes greater than the antenna half-power beam width $[10,11]$.

\section{KALMAN FILTER ENHANCEMENT ON THE MISSILE TARGET ENGAGEMENT}

A six-state Kalman filter is utilized to perform the tracking task and perform smoothing to the corrupted trajectory. For each time step during the missile target engagement, the unfiltered position of the target $\left(X_{T_{I}}, Y_{T_{I}}, Z_{T_{I}}\right.$ ) is filtered through a Kalman filter before putting it again into algorithm to get the responding missile position.

The enhancement effect of using the Kalman estimator in the missile target tracking algorithm is described as follows, first we calculate the filtered position of the target $X_{T_{F_{(i t 1)}}}, Y_{T_{F_{(i t}+1}}, Z_{T_{F_{(i t+1}}}$ by injecting the unfiltered target position $X_{T_{I_{(i+1)}}}, Y_{T_{I_{(i+1)}}}, Z_{T_{I_{(i+1)}}}$ to Kalman to be filtered and also the missile unfiltered position $X_{M_{I_{(i)}}}, Y_{M_{I_{(i)}}}, Z_{M_{I_{(i)}}}$ to get the filtered position $X_{M_{F(i)}}, Y_{M_{F(i)}}, Z_{M_{F(i)}}$, next the filtered LOS angles $\varepsilon_{F_{(i+1)}}, \sigma_{F_{(i+1)}}$ can be calculated from the following equations 


$$
\begin{gathered}
\varepsilon_{F i+1}=\tan ^{-1}\left(\frac{Y_{T_{F i+1}}-Y_{M_{F_{i}}}}{\sqrt{\left(X_{T_{F_{i+1}}}-X_{M_{F_{i}}}\right)^{2}+\left(Z_{T_{F_{i+1}}}-Z_{M_{F_{i}}}\right)^{2}}}\right) \\
\sigma_{F i+1}=\tan ^{-1}\left(\frac{Z_{T_{F_{i+1}}}-Z_{M_{F_{i}}}}{X_{T_{F i+1}}-X_{M_{F_{i}}}}\right)
\end{gathered}
$$

where these filtered angles are used by the missile to set his LOS angles in azimuth and elevation respectively, and to calculate the next filtered position of the missile towards the target.

\section{SIMULATION RESULTS}

In section 6.1, we will simulate a 3-dimensional scenario between target (with high manoeuvre) and the missile in the case when no interference is present. When interference is present, it is shown in section 6.2. The Kalman improvement for the tacking for the three guidance method will be analysed in section 6.3.

For all the previous simulations algorithms, we consider 3-dimensional motion and apply the kinematics equations (Eq.10) together with the law of guidance equations. The following initial conditions are used for the missile target engagement as shown previously in Fig 1, the initial LOS length $D_{o}=10 \mathrm{~km}$, the initial LOS angles $\varepsilon_{o}=20^{\circ}, \sigma_{o}=20^{\circ}$, the missile velocity $V_{M}=800 \mathrm{~km} / \mathrm{hour}$, the target velocity $V_{T}=600 \mathrm{~km} / \mathrm{hour}$, and the initials angles of the target's velocity $\theta_{T}=40^{\circ}, \varphi_{T}=30^{\circ}$ and the corresponding calculated missile velocity angles (calculated from Eq.10) $\theta_{M}=33.34^{\circ}, \varphi_{M}=26.86^{\circ}$

\subsection{Target-missile under different guidance methods}

In Fig 2, one target trajectory is simulated in black. It performs a hard manoeuvre with the target parameters mentioned in section 6 . The corresponding three missile trajectories due to the three guidance methods (blue, pink, and red trajectories) are plotted. It is seen that each missile follows the target in different ways according to the guidance bond equations and as a result will meet the target at different points in the space. The length and shape of the missile trajectory depends also on the guidance method as well as the time of flight to the hit point. The LOS distance will vary with time while the flight time also will depend on the guidance method. In this section we simulated a 3dimensional scenario between target and corresponding three missile trajectories in the case when no interference is present.

\subsection{Target-missile with the effect of interference}

The ability of the missile seeker to determine the target position is affected by different types of interferences (clutter, thermal noise, jamming) [3]. Consequently the target trajectory will suffer from that error. For simulation purposes, the target trajectory is corrupted by adding white Gaussian

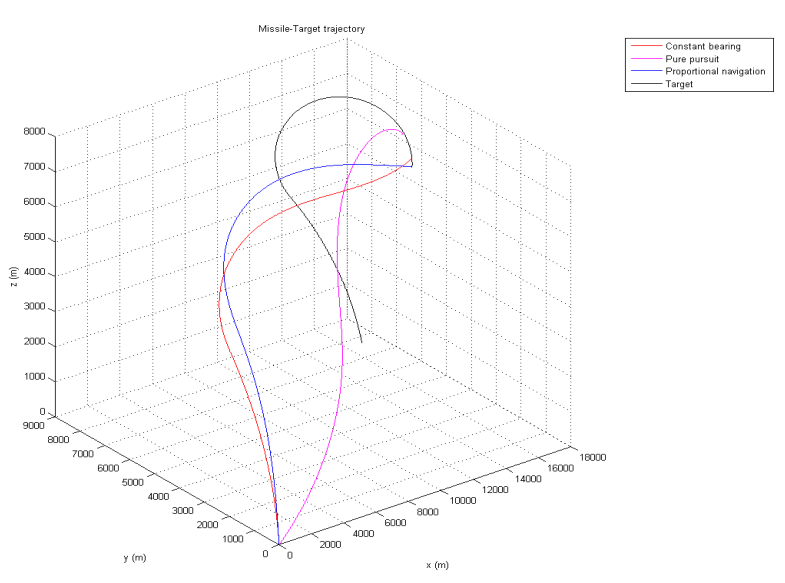

Fig 2. The target-missile trajectories

noise to it with the considered standard deviation of azimuth error $=.008$ radians, standard deviation of elevation error $=.009$ radians, and standard deviation of range error $=1 \mathrm{~m}$ [7] Five seconds after the missile firing, we start to corrupt the missile- target trajectory to increase the simulation reality as shown in Fig 3 (a, b, and c) which illustrates the filtered portion of the target path.

In the simulation algorithm, the missile processor calculates LOS angles under interference $\varepsilon_{I_{(i+1)}}, \sigma_{I_{(i+1)}}$ from equations 11 and 12 along his flight. This process is repeated until the difference between the angles $\varepsilon_{l_{i}}, \varepsilon_{l_{t+1}}$ becomes greater than the antenna half-power beam width which considered in the simulation equal $8^{\circ}$. At this instant the missile seeker loses the target, which is out of its field of view is part of the missile trajectory is shown in Fig 3 (a, b, and c) as the green line for the three guidance method. The effect of interference on the missile target trajectory appears in the simulation as a slight drift of the missile position because it follows the unfiltered target trajectory. This drift will increase in the final course of the missile flight so at a particular point the missile can't keep tracking and it will lose the target.

The points of miss tracking depend on the guidance method and the effect of interference on the seen target position from the missile point of view. The target senses the instant it is outside the radar, and then the target pilot can start manoeuvring away from the missile. Usually the missile guidance and control systems include a memory device, such that if the seeker loses tracking the target, it will keep the final value of its velocity vector angle.

$$
\begin{aligned}
& \theta_{M_{t(t)+}}=\theta_{M_{t,}} \\
& \varphi_{M_{t+1}}=\varphi_{M_{t}}
\end{aligned}
$$

If the fuse system in the missile dose not sense the target for a certain time (equals $30 \mathrm{sec}$. in the simulations), the missile will self destroy. In Fig 3 (a, b, and c) this situation is simulated using the final data from the missile flight and we also assume that the aeroplane will keep its angular velocity after the point of miss tracking. 


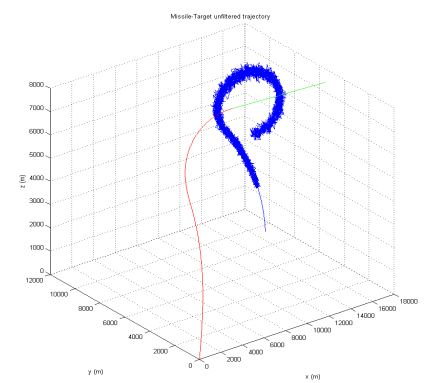

(a)

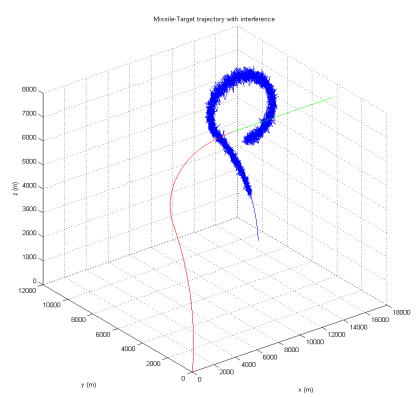

(b)

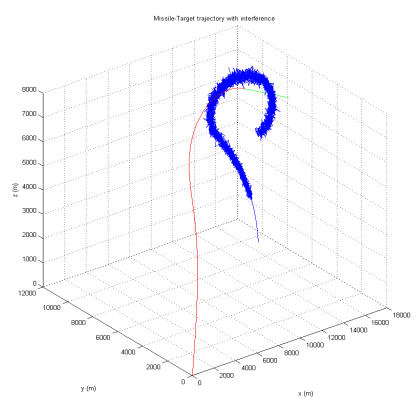

(c)

Fig 3. Unfiltered trajectory

(a) proportional navigation

(b) constant bearing

(c) pure pursuit

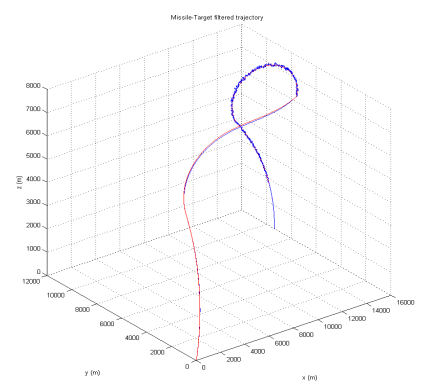

(a)

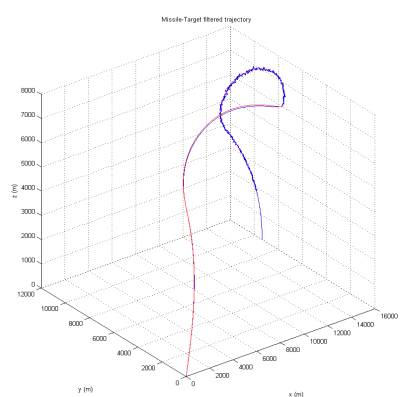

(b)

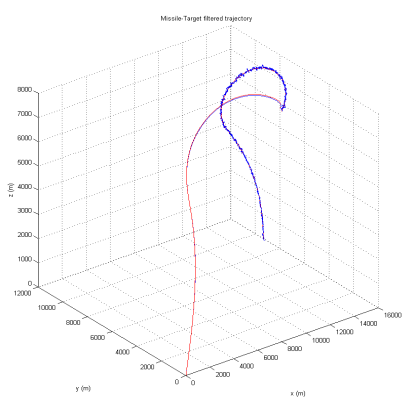

(c)

Fig 4. Filtered trajectory

(a) proportional navigation

(b) constant bearing

(c) pure pursuit

\subsection{Target-missile with the effect of interference using Kalman filter}

In the simulation algorithm, the missile processor with Kalman capabilities is shown in Fig 4 ( $a, b$, and c). The simulated filtered missile target trajectories are shown in blue while the ideal missile target trajectories are shown in red. The missile processor calculates the filtered LOS angles $\varepsilon_{F_{(i+1)}}, \sigma_{F_{(i+1)}}$ from equations 13 and 14 along the missile flight as shown in Fig 4 (a, b, and c). It is clear that the filtered trajectories for all three guidance methods follows the target and the difference between the missile in the filtered case and the ideal target is less than the fusing distance of the missile. This is continued until the line of site (LOS) distance between the filtered missile position and the target equals zero or equal the fusing distance of the missile, typically considered $50 \mathrm{~m}$.

\section{CONCLUSION}

In this paper we have described the effect that interference has on the missile seeker. Introducing interference into the tracking radar system decreases the tracker ability to determine the exact target position. From the radar point of view it will corrupt the target location and consequently drive the missile to an incorrect location for the three guidance methods and this leads to target miss tracking.

The Kalman filter as a linear estimator was used in this paper to minimize the mean squared error of the LOS tracking angle due to interference. It is not a method to decrease or cancel this interference, but makes successive improvement of the missile's ability to keep tracking the corrupted target trajectory until the LOS distance reaches the fusing distance (hit the target). In our future work we will focus on how to minimize or cancel this interference especially resulting from clutter and terrain scattering interference.

\section{REFRENCES}

[1] R. Klemm and U. Nickel, "Adaptive monopluse with STAP," in Radar, 2006. CIE '06. International Conference on, 2006, pp. 1-4.

[2] M. I. Skolnik, Radar Handbook- Second edition: McGraw-Hill, Inc., 1990.

[3] A. D. Seifer, "Monopulse-radar angle tracking in noise or noise jamming," Aerospace and Electronic Systems, IEEE Transactions on, vol. 28, pp. 622-638, 1992.

[4] H. Meikle, Modern Radar Systems Artech House, Inc., 2008.

[5] Y. Seliktar, E. J. Holder, and D. B. Williams, "An adaptive monopulse processor for angle estimation in a mainbeam jamming and coherent interference scenario," in Acoustics, Speech and Signal Processing, 1998. Proceedings of the 1998 IEEE International Conference on, 1998, pp. 2037-2040 vol.4.

[6] B. R. Mahafza, Radar system analysis and design using Matlab: Boca Raton, FL: CRC Press/Chapman \& Hall, 2005.

[7] B. R. Mahafza and A. Z. Elsherbeni, MATLAB simulations for radar systems design: Boca Raton, FL: CRC Press/Chapman \& Hall, 2004.

[8] S. A. Elgamel and A. H. Makaryous, "Performance of Modern Guided Systems in Presence of Jamming Signal ," in Aerospace Science and Aviation Technology, The 9th International conference on, , Cairo, Egypt, 2001.

[9] M. R. M. Rizk and M. A. Rahim, "Robust stochastic controller for a missile guidance system," in Electronics, Circuits and Systems, 2000. ICECS 2000. The 7th IEEE International Conference on, 2000, pp. 793-796 vol.2.

[10] M. I. Skolnik, Radar Handbook-Third edition: McGrawHill, Inc., 2008.

[11] Y. Seliktar, E. J. Holder, and D. B. Williams, "A space/fast-time adaptive monopulse technique." vol. 2006: Hindawi Publishing Corp., pp. 218-218. 